\title{
Factores sociodemográficos asociados a la satisfacción con los servicios públicos de salud entre personas con $\mathrm{VH} /$ SIDA $^{*}$
}

\section{Sociodemographic correlates of satisfaction with public health services among people living with HIV}

\section{Fatores sociodemográficos associados à satisfação com os serviços públicos de saúde entre pessoas com VIH/SIDA}

Fecha de recepción: 30-09-13 Fecha de aceptación: 05-03-14

doi:10.11144/Javeriana.RGYPS13-26.fsas

\author{
María Sandra Compean-Dardón** \\ Diana Pérez-Salgado***
}

Mtra. María Guadalupe Staines-Orozco****

Luis Ortiz-Hernández $* * * * *$

Artículo de investigación. Este trabajo es producto del proyecto Factores asociados a la no adherencia al tratamiento antirretroviral en personas con VIH/SIDA. Aunque se trata de un proyecto en progreso, los datos reportados aquí fueron recabados de octubre del 2011 a noviembre de 2012. El proyecto fue sancionado por la Universidad Autónoma Metropolitana unidad Xochimilco y recibió financiamiento del Programa de Mejoramiento del Profesorado (PROMEP) de la Secretaría de Educación Pública. Dirección de correspondencia: Luis Ortiz Hernández Calzada del Hueso 1100, Col. Villa Quietud, Coyoacán, México, 04960. Tel.: (+52) (55) 54-83-72-43, Fax: (+52) (55) 54-83-72-18. Correo electrónico: lortiz@correo.xoc.uam.mx

** Maestra en Salud Pública, profesora investigadora en el Departamento de Atención a la Salud, Universidad Autónoma Metropolitana unidad Xochimilco. Correo electrónico: sandrac_d@hotmail.com

*** Licenciada en Nutrición, asistente de investigación en el Departamento de Atención a la Salud, Universidad Autónoma Metropolitana unidad Xochimilco. Correo electrónico: dianaps2006@gmail.com

***** Maestra en Didáctica y Conciencia Histórica. Técnica académica en el Departamento de Atención a la Salud, Universidad Autónoma Metropolitana unidad Xochimilco. Correo electrónico: gstaineso@yahoo.com

***** Doctor en Ciencias en Salud Colectiva, profesor investigador en el Departamento de Atención a la Salud, Universidad Autónoma Metropolitana unidad Xochimilco. Correo electrónico: lortiz@correo.xoc.uam.mx 


\section{Resumen}

Objetivo: evaluar la satisfacción de personas con VIH con la atención que reciben en los servicios de salud. Material y métodos: se evaluó la satisfacción del usuario y las características sociodemográficas de personas con VIH/SIDA atendidas en dos instituciones públicas de la ciudad de México $(n=557)$. Para conocer si existían diferencias se estimaron modelos de regresión logística. Resultados: los hombres homosexuales tuvieron mayor probabilidad de insatisfacción con la relación médico-paciente y con el funcionamiento del servicio. Las personas con seguridad social tuvieron mayor probabilidad de insatisfacción con el funcionamiento del servicio y con la atención de las diferentes áreas de la clínica. Los hombres, las personas solteras y quienes tenían mayor escolaridad presentaron mayor insatisfacción con los aspectos administrativos. Conclusiones: se identificaron aspectos de los servicios públicos que pueden ser mejorados, lo cual podría modificar la opinión que las personas con vIH tuvieron sobre ellos.

Palabras clave: satisfacción del paciente; servicios de salud; vIH; SIDA; México; características de la población

Palabras clave descriptor: satisfacción del paciente, servicios de salud, vIH, síndrome de inmunodeficiencia adquirida- Mexico

\section{Abstract}

Objectives: To evaluate the satisfaction of people with HIV/AIDS with the care received from public health services. Material and methods: The user satisfaction and socio-demographic characteristics of people with HIV/AIDS who were treated at two public institutions in Mexico City $(n=557)$ were assessed. To know whether there were any differences, logistic regression models were estimated. Results: Homosexual males were more likely to be dissatisfied with physician-patient relationship and with services operation. People with social security had higher probability of dissatisfaction with service operation and with the attention received from different areas. Dissatisfaction with administrative aspects was more frequent among men, single people and those who had higher education higher. Conclusions: There were aspects of public services that can be improved, which could result in the improvement of the opinion that people with HIV had of them.

Keywords: patient satisfaction, health services, HIV, AIDS, Mexico, population characteristics Keywords plus: patient satisfaction, health services, HIV, adquired immunodeficiency syndrome- Mexico

\section{Resumo}

Objetivo: avaliar a satisfação de pessoas com o VIH, atendidos em serviços de saúde. Material e métodos: avaliou-se a satisfação do utente e as características sociodemográficas do pessoal com o VIH/SIDA atendidas em duas instituições públicas da cidade de México ( $\mathrm{n}=557)$. Para conhecer se existiam diferenças foram estimados modelos de regressão logística. Resultados: os homes homossexuais tiveram maior probabilidade de insatisfação na relação médico-paciente e com o funcionamento do serviço. As pessoas com previdência social tiveram maior probabilidade de insatisfação com o funcionamento do serviço e a atenção das diferentes áreas da clínica. Os homes, as pessoas solteiras e quem teve maior escolaridade apresentou maior insatisfação com os aspetos administrativos. Conclusões: identificaram-se aspectos dos serviços públicos que podem se melhorar, o qual poderia modificar a opinião das pessoas com VIH. .

Palavras chave: satisfação do paciente, serviços de saúde, VIH, SIDA, México, características da população

Palavras chave descritores: satisfação do paciente, serviços de saúde, VIH, síndrome de inmunodeficiência adquirida - México 


\section{Introducción}

La infección por VIH sigue siendo uno de los problemas de salud más graves a nivel mundial. A finales de 2011, 34 millones de personas vivían con el VIH en todo el mundo (1). En México de 1983 a 2012 se diagnosticaron 160.864 casos y durante 2011 murieron 5043 personas por causas relacionadas con el SIDA (2). El mayor porcentaje de casos notificados de SIDA ha sido entre los hombres $(82,1 \%)$, particularmente entre los que tienen de 30 a 39 años de edad. Tomando en cuenta a ambos sexos, los grupos de edad más afectados son los de 25 a 44 años.

Por su condición crónica, las personas que viven con VIH deben acudir constantemente a las instituciones de salud en donde reciben su tratamiento. Debido a la interacción continua con los servicios de salud, resulta relevante conocer los factores que pueden influir en la colaboración de los pacientes con el manejo de su enfermedad. Uno de esos factores puede ser la calidad de los servicios de salud.

Este trabajo se centra en la aceptabilidad de los servicios de salud para el paciente, que es una de las seis dimensiones que la Organización Mundial de la Salud (oms) (3) considera para evaluar la calidad de los sistemas y servicios de salud. Un método para evaluar la aceptabilidad de la atención de los servicios y proveedores de salud es la satisfacción de las personas que son atendidas en las instituciones de salud (4,5). Los índices de satisfacción buscan una evaluación personal de los servicios de salud que no puede ser medida por observación directa de estos (4). Es decir, se trata de una evaluación subjetiva de los usuarios que algunas veces no va a corresponder con la percepción de los proveedores y administradores de la atención. Por tanto, con los índices de satisfacción se puede recabar información única respecto a la calidad de los servicios de salud que sería difícil obtener mediante otras metodologías.

Los estudios relacionados con la satisfacción del usuario permiten integrar los puntos de vista del paciente respecto al funcionamiento del sector salud e identificar problemas y dificultades en los servicios de salud. El tomar en cuenta la perspectiva de los usuarios puede tener un impacto en el manejo y solución de los problemas identificados dentro de los servicios (6).

La satisfacción del paciente refleja las experiencias, expectativas y preferencias respecto a la accesibilidad del cuidado médico, la disponibilidad de los servicios de salud, la asequibilidad de los costos, la información otorgada por el personal de salud y la participación del paciente (i.e., integración del paciente en la decisión de los procedimientos y en el tratamiento) $(6,7)$. De acuerdo con algunos estudios, la satisfacción del usuario puede influir en su conducta, por ejemplo en el apego al tratamiento $(8,9)$ y la continuidad en el uso de los servicios de salud (10). Específicamente, una relación médico-paciente positiva se ha vinculado a mayor adherencia al tratamiento y mejora en los resultados clínicos de los pacientes (11).

En México algunos estudios han identificado los aspectos que integran la satisfacción con los servicios de salud (12) y han evaluado la satisfacción de los usuarios con la atención de algunas instituciones públicas. Algunos autores han descrito la satisfacción con los servicios en general de toda una clínica (13) y de áreas específicas de una unidad de salud (i. e., servicios de atención en el trabajo, atención prenatal) $(14,15)$. También se ha evaluado la satisfacción en poblaciones particulares (e. g., las personas con enfermedad crónica) (16). Sin embargo, existe poca información respecto a la satisfacción de personas que viven con VIH/SIDA con los 
servicios recibidos en instituciones públicas. A nivel internacional también es escasa la evidencia al respecto pues solo identificamos un estudio realizado en Vietnam (17).

En México, la Ciudad de México es la entidad que concentra el mayor número de casos de VIH. En este país existe un programa de acceso universal a la atención médica para las personas que viven con VIH que incluye, particularmente, el acceso a los antirretrovirales. En la Ciudad de México, las personas con VIH que cuentan con seguridad social son atendidas en las instituciones correspondientes: el Instituto de Seguridad y Servicios Sociales de los Trabajadores del Estado o ISSSTE para trabajadores gubernamentales y el Instituto Mexicano del Seguro Social o IMSS para trabajadores de empresas privadas. Las personas que no tienen acceso a seguridad social pueden ser atendidas en las Secretarías de Salud (ss) de los gobiernos estatales o del Distrito Federal.

A nivel nacional el 77\% de los casos de VIH/ SIDA son atendidos por las ss, el 19,9\% por el IMSS y el 3,0\% por el ISSSTE (18). En la Ciudad de México la ss local tiene un centro de primer nivel especializado en VIH/SIDA llamado Clínica Condesa que es el centro de referencia más grande del país. En el año 2011 atendió a 5449 personas con VIH. El ISSSTE cuanta con clínicas de VIH en sus hospitales de segundo y tercer nivel. El hospital del ISSSTE donde se realizó el estudio -Hospital Regional Lic. Adolfo López Mateos- es un centro de atención de tercer nivel que atiende a más de 300 pacientes.

Por todo lo anterior, los objetivos de este trabajo fueron: 1) evaluar la satisfacción de personas seropositivas con los servicios para la atención del VIH/SIDA de dos instituciones públicas de salud y 2) identificar los facto130 res sociodemográficos relacionados con la satisfacción.
Conflicto de intereses. Los autores declaran no tener conflicto de intereses en relación con este trabajo.

\section{Material y métodos}

El proyecto de investigación del cual se deriva el presente trabajo fue aprobado por el Consejo Divisional de Ciencias Biológicas y de la Salud de la Universidad Autónoma Metropolitana unidad Xochimilco. La revisión de los proyectos de investigación por dicho consejo incluye los aspectos éticos.

Se realizó un estudio transversal analítico y comparativo. Se obtuvo una muestra por conveniencia de las personas seropositivas atendidas dos instituciones de salud de la Ciudad de México: 1) la clínica de VIH del Hospital Regional "Lic. Adolfo López Mateos" del Issste y 2) la Clínica Especializada Condesa de la Secretaría de Salud del Gobierno de Distrito Federal (ss-GDF). Se han seleccionado estos centros pues representan diferentes tipos de instituciones y, por tanto, de poblaciones: un centro de atención de alta especialidad de la seguridad social y un centro donde se atiende a población abierta (i. e. sin seguridad social). En comparación con las instituciones dirigidas a población abierta, es previsible que en las instituciones de seguridad social la calidad de atención sea mejor y que tengan menos restricciones presupuestales. Además, las personas que acuden a estas instituciones son, en promedio, de posición socioeconómica más alta.

El trabajo de campo del presente estudio se realizó de octubre de 2011 a noviembre de 2012. Los criterios de inclusión fueron: ser usuario de alguna de las dos clínicas, estar bajo tratamiento antirretroviral, ser mayor de 18 años o, en el caso de los menores de edad, que ya no dependieran de sus tutores ni vivieran con ellos. Se obtuvo 
el consentimiento informado por escrito de cada participante. La muestra total estuvo conformada por 557 personas de entre $16 \mathrm{y}$ 80 años. La aplicación de los cuestionarios fue realizada por pasantes de la Licenciatura en Enfermería previamente capacitadas en técnicas de entrevista.

Se aplicó un cuestionario para registrar información relacionada con la satisfacción del usuario con los servicios de la clínica en donde era atendido así como sus características sociodemográficas. La variable dependiente fue la satisfacción con la atención de los servicios de salud y fue evaluada utilizando 17 preguntas de una escala validada en México en personas con enfermedades crónicas (16). Además, se elaboró una nueva escala de 5 preguntas para evaluar la satisfacción con la atención recibida en áreas específicas de los servicios (laboratorio, farmacia, enfermería, recepción y trabajo social).

Para ambas escalas se pidió a los participantes que contestaran cada pregunta utilizando una escala tipo Likert con cinco opciones de respuestas: nada satisfecho, poco satisfecho, satisfecho, muy satisfecho y completamente satisfecho. A las opciones se les asignó una puntuación de uno a cinco, respectivamente. Se realizó el análisis factorial exploratorio para identificar los índices de satisfacción. Cada índice (factor) fue integrado por todas las preguntas que tenían un peso $>0,40$ (19). Mediante el análisis factorial exploratorio se identificaron tres componentes o factores para la primera escala y un componente para la segunda (ver tabla 1).

De la primera escala, el primer componente evalúa la satisfacción con la relación médicopaciente y se integró con nueve ítems: amabilidad del médico, examen físico, tratamiento, información del médico respecto a los efectos secundarios del tratamiento, si el médico permite al paciente dar su opinión acerca del tratamiento, aclaración de las dudas del paciente respecto al tratamiento, si el paciente entiende la información proporcionada por el médico, tiempo de la consulta y satisfacción general con el médico. El segundo factor evalúa la satisfacción con el funcionamiento del servicio y se conformó por cuatro ítems: frecuencia con que le proporcionan todas las medicinas prescritas, comodidad de la clínica, limpieza de la clínica y confianza del paciente en la clínica. El tercer factor evalúa los aspectos administrativos del servicio y se formó por cuatro ítems: organización del hospital, horarios de las citas asignadas, resolución de problemas administrativos de la clínica y tiempo de espera para pasar a consulta.

De la segunda escala solo emergió un componente que evalúa la satisfacción con atención de las diferentes áreas de la clínica: atención del laboratorio, atención en la farmacia, atención del servicio de enfermería, atención en la recepción y atención en trabajo social.

Se calculó el puntaje promedio de todas las preguntas en cada componente, es decir, se sumaron las puntuaciones de las respuestas y se dividió por el número de preguntas incluidas en el factor o componente. Después cada factor fue dividido en dos categorías: satisfacción e insatisfacción. Se consideró que un participante estaba satisfecho cuando el puntaje promedio para cada índice era $\geq 3,0$ e insatisfecho cuando el puntaje promedio era $<3,0$, lo cual corresponde con los valores asignados a las categorías de respuesta (i. e., las opciones de respuesta "satisfecho", "muy satisfecho" y "completamente satisfecho" tienen puntuaciones de 3 , 4 y 5 , respectivamente).

Las variables independientes fueron la edad, el sexo, el estado civil, la orientación sexual (la cual se indagó solo en los hombres), la ocupación del participante, la escolaridad 


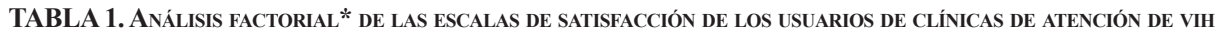

\begin{tabular}{|c|c|c|c|}
\hline & $\begin{array}{l}\text { Factor } 1 \\
\text { Relación } \\
\text { médico- } \\
\text { paciente }\end{array}$ & $\begin{array}{l}\text { Factor } 2 \\
\text { Funciona- } \\
\text { miento del } \\
\text { servicio }\end{array}$ & $\begin{array}{l}\text { Factor } 3 \\
\text { Aspectos } \\
\text { administra- } \\
\text { tivos }\end{array}$ \\
\hline \multicolumn{4}{|l|}{ Escala tomada de Vladislavovna-Doubova et ál. (14) } \\
\hline \multicolumn{4}{|l|}{$\begin{array}{l}\text { Porcentaje de la varianza } \\
\text { Eigen-value }\end{array}$} \\
\hline 1. La organización del hospital-clínica & 0,15 & 0,14 & 0,85 \\
\hline 2. Los horarios respecto a sus citas asignadas & 0,15 & 0,16 & 0,87 \\
\hline $\begin{array}{l}\text { 3. La facilidad que se resuelven los problemas administrativos de } \\
\text { su hospital o clínica }\end{array}$ & 0,22 & 0,15 & 0,85 \\
\hline $\begin{array}{l}\text { 4. El tiempo de espera desde el momento de su cita hasta que lo } \\
\text { pasan a consulta }\end{array}$ & 0,13 & 0,29 & 0,69 \\
\hline $\begin{array}{l}\text { 5. La frecuencia con que le dan todas las medicinas prescritas en } \\
\text { la farmacia del hospital o la clínica }\end{array}$ & 0,25 & 0,70 & 0,26 \\
\hline 6. La comodidad del hospital o la clínica & 0,29 & 0,84 & 0,25 \\
\hline 7. La limpieza del hospital o la clínica & 0,28 & 0,88 & 0,15 \\
\hline 8. La confianza que tiene en el hospital o clínica & 0,33 & 0,85 & 0,16 \\
\hline 9. La amabilidad del médico & 0,62 & 0,52 & 0,22 \\
\hline 10. La exploración física que el médico le realiza & 0,66 & 0,44 & 0,25 \\
\hline 11. El tratamiento que le da el médico por su enfermedad & 0,68 & 0,43 & 0,27 \\
\hline $\begin{array}{l}\text { 12. La información que el médico le da sobre los efectos } \\
\text { secundarios que puedan surgir de los medicamentos durante su } \\
\text { tratamiento }\end{array}$ & 0,83 & 0.27 & 0,20 \\
\hline 13. Poderle dar al médico su opinión acerca de su tratamiento & 0,87 & 0,28 & 0,16 \\
\hline $\begin{array}{l}\text { 14. Las aclaraciones del médico a sus dudas acerca del } \\
\text { tratamiento }\end{array}$ & 0,90 & 0,25 & 0,12 \\
\hline 15. Que tan bien entiende la información que le brinda el médico & 0,89 & 0,23 & 0,15 \\
\hline 16. El tiempo que le dedica el médico en la consulta & 0,91 & 0,19 & 0,14 \\
\hline 17. En general está satisfecho(a) con su médico & 0,88 & 0,17 & 0,11 \\
\hline Escala desarrollada & \multicolumn{2}{|c|}{$\begin{array}{l}\text { Factor } 1 \\
\text { Atención en servicios } \\
\text { específicos }\end{array}$} & \\
\hline \multicolumn{4}{|l|}{$\begin{array}{l}\text { Pocentaje de la varianza } \\
\text { Eigen-value }\end{array}$} \\
\hline 1. La atención que proporciona el laboratorio & 0,82 & & \\
\hline 2. La atención que proporciona la farmacia & 0,88 & & \\
\hline 3. La atención por parte del servicio de enfermería & 0,89 & & \\
\hline 4. La atención por parte de la recepción & 0,88 & & \\
\hline 5. La atención por parte de trabajo social & 0,83 & & \\
\hline
\end{tabular}

*Análisis con rotación varimax.

Fuente: elaboración propia con datos recabados en dos instituciones públicas de la Ciudad de México 
del jefe de familia y la institución en donde era atendido. Los grupos de edad fueron: a) 16 a 25, b) 26 a 35, c) 36 a 50 y d) 50 y más años. Para el estado civil se conformaron tres grupos: a) soltero, b) casado o en unión libre y c) divorciado o viudo.

En orientación se formaron tres grupos: 1) heterosexual (se incluyó la opción "heterosexual solamente"), 2) bisexual (se incluyeron las opciones "bisexual, pero más heterosexual", "bisexual" y "bisexual pero más homosexual”), y 3) homosexual (opción "homosexual solamente"). En la ocupación del participante las opciones de respuesta eran: no trabaja, trabajador de empresa privada, trabajador de Gobierno, trabaja para otra persona, trabaja por su cuenta o empresario, patrón, empleador o dueño. En cuanto a la escolaridad del jefe de familia (i. e. persona que sostenía económicamente el lugar donde vive el entrevistado), se establecieron cuatro grupos: 1) primaria o menos, 2) secundaria, 3) bachillerato o carrera técnica y 4) licenciatura y más.

Se realizó el análisis descriptivo de las variables en el cual se comparó la frecuencia de satisfacción entre los grupos de las variables independientes. Para determinar las características de los participantes que estaban asociadas con cada factor, se realizó el análisis bivariado usando la prueba de chi cuadrado para conocer si existían diferencias estadísticamente significativas $(p \leq 0,050)$. Posteriormente, se realizaron modelos de regresión logística para obtener la asociación ajustada entre las características de los participantes y la insatisfacción en cada una de las dimensiones (relación médico-paciente, funcionamiento del servicio, aspectos administrativos y con atención en áreas específicas). Este procedimiento se realizó para la población total y para cada sexo. Solo se presentan los resultados de la población total y de los hombres, ya que en ellos existieron asociaciones. La variable de orientación sexual se utilizó en el análisis que incluía únicamente a los hombres porque solo en ellos se indagó dicho aspecto. El análisis estadístico se llevó a cabo en el programa spss versión 19.0.

\section{Resultados}

La media de edad fue de 36 años en la población total y fue mayor entre los usuarios de la clínica del issste. En ambas instituciones la mayoría fueron hombres y la mayoría eran solteros $(62,3 \%)$, seguidos por los que estaban casados o en unión libre $(26,2 \%)$ (ver tabla 2). Casi existió la misma proporción de personas que trabajaban por su cuenta $(26,2 \%)$ que de personas que no trabajaban $(25,5 \%)$, seguidos por los que eran trabajadores de empresas privadas $(17,2 \%)$. En cuanto a la escolaridad del jefe de familia, el mayor porcentaje $(38,7 \%)$ tenía como último grado de estudios el bachillerato, seguidos por los que tenían la secundaria $(25,5 \%)$. En los hombres, respecto a la orientación sexual, el 57,1\% manifestó ser homosexual, el $24,8 \%$ heterosexual y el $17,9 \%$ bisexual.

La media de satisfacción de los participantes con la relación médico-paciente fue de 3,84 puntos (ver tabla 3). Los rubros que tuvieron mayor puntaje fueron "la amabilidad del médico" y "la satisfacción general con el médico", mientras que los tuvieron el menor puntaje fueron "la información que el médico otorga sobre los efectos secundarios del tratamiento", "la exploración física que realiza el médico" y "poder dar su opinión al médico acerca de su tratamiento".

El puntaje promedio respecto al funcionamiento del servicio fue de 3,71; el puntaje más alto fue para "la confianza que tiene en la clínica" y los más bajos para "la frecuencia con que le dan todas las medicinas prescritas en la 
TABLA 2. Características SOCIODEMOGRÁFiCas de la POBLACión

\begin{tabular}{|c|c|c|c|c|c|c|}
\hline & \multicolumn{2}{|c|}{ Total } & \multicolumn{2}{|c|}{ ISSSTE } & \multicolumn{2}{|c|}{ SS-GDF } \\
\hline \multirow[t]{2}{*}{ Edad (media) } & \multicolumn{2}{|c|}{36,6} & \multicolumn{2}{|c|}{48,2} & \multicolumn{2}{|c|}{34,0} \\
\hline & $\mathrm{N}$ & $\%$ & $\mathrm{~N}$ & $\%$ & $\mathrm{~N}$ & $\%$ \\
\hline \multicolumn{7}{|l|}{ Sexo } \\
\hline Hombres & 448 & 80,4 & 83 & 83,0 & 365 & 79,9 \\
\hline Mujeres & 109 & 19,6 & 17 & 17,0 & 92 & 20,1 \\
\hline \multicolumn{7}{|l|}{ Estado civil } \\
\hline Soltero & 347 & 62,3 & 50 & 50,0 & 297 & 65,0 \\
\hline Casado-unión libre & 146 & 26,2 & 33 & 33,0 & 113 & 24,7 \\
\hline Divorciado-viudo & 64 & 11,5 & 17 & 17,0 & 47 & 10,3 \\
\hline \multicolumn{7}{|l|}{ Orientación sexual * } \\
\hline Heterosexual & 111 & 24,8 & 46 & 55,4 & 65 & 17,8 \\
\hline Bisexual & 80 & 17,9 & 4 & 4,8 & 76 & 20,8 \\
\hline Homosexual & 256 & 57,1 & 33 & 39,8 & 223 & 61,0 \\
\hline No está seguro & 1 & 0,2 & & & 1 & 0,3 \\
\hline \multicolumn{7}{|l|}{ Ocupación } \\
\hline No trabaja & 142 & 25,5 & 21 & 21,0 & 121 & 26,5 \\
\hline Trabajador de empresa privada & 96 & 17,2 & 4 & 4,0 & 92 & 20,1 \\
\hline Trabajador de gobierno & 79 & 14,2 & 71 & 71,0 & 8,0 & 1,8 \\
\hline Trabaja para otra persona & 71 & 12,7 & 0 & 0 & 71 & 15,5 \\
\hline Trabaja por su cuenta & 146 & 26,2 & 4 & 4,0 & 142 & 31,1 \\
\hline Empresario, patrón, empleador o dueño & 23 & 4,2 & 0 & 0 & 23 & 5,0 \\
\hline \multicolumn{7}{|l|}{ Escolaridad del jefe de familia } \\
\hline Primaria o menos & 74 & 13,3 & 6 & 6,1 & 68 & 14,9 \\
\hline Secundaria & 142 & 25,5 & 6 & 6,1 & 136 & 29,8 \\
\hline Bachillerato & 215 & 38,7 & 33 & 33,3 & 182 & 39,8 \\
\hline Licenciatura y más & 125 & 22,5 & 54 & 54,5 & 71 & 15,5 \\
\hline
\end{tabular}

* Esta pregunta solo fue aplicada a los varones.

Abreviaturas: ISsSTE: Instituto de Seguridad y Servicios Sociales de los Trabajadores del Estado. SS-GDF: Secretaría de Salud del Gobierno de Distrito Federal.

Fuente: elaboración propia con datos recabados en dos instituciones públicas de la Ciudad de México

farmacia de la clínica" y "la comodidad de la clínica". La media de satisfacción con los aspectos administrativos fue de 3,35 ; el puntaje más alto fue para "la organización general del hospital", mientras que el más bajo fue para "el tiempo de espera en pasar a consulta". En cuanto a la atención de las diferentes áreas de la clínica. el puntaje promedio fue de 3,78, siendo la atención de enfermería la que tuvo el puntaje más alto y la atención de trabajo social la que tuvo el más bajo.

$\mathrm{Al}$ categorizar a los participantes en satisfechos e insatisfechos se observó que 10,8\% estuvieron insatisfechos con la relación médico-paciente; $12,8 \%$ con el funcionamiento del servicio, $32,5 \%$ con los aspectos 
FACTORES SOCIODEMOGRÁFICOS ASOCIADOS A LA SATISFACCIÓN CON LOS SERVICIOS PÚBLICOS DE SALUD ENTRE PERSONAS CON VIH/SIDA

TABLA 3. Satisfacción los usuarios de Clínicas de atención de VIH CON La RELACión MÉdico-PaCiente, EL HOSPITAL O CLÍNICA, LA ORGANIZACIÓN DE LA CLÍNICA Y LA ATENCIÓN

\begin{tabular}{|c|c|}
\hline & $\begin{array}{c}n=557 \\
\text { Puntaje prome- } \\
\text { dio } \pm \text { DE }\end{array}$ \\
\hline \multicolumn{2}{|l|}{ Factores } \\
\hline I. Relación médico-paciente & $3,84 \pm 0,84$ \\
\hline Amabilidad del doctor & $3,95 \pm 0,96$ \\
\hline Exploración física que el médico le realiza & $3,81 \pm 0,99$ \\
\hline Tratamiento que le da el médico por su enfermedad & $3,93 \pm 0,96$ \\
\hline Información que el médico otorga sobre efectos secundarios de los medicamentos & $3,82 \pm 0,99$ \\
\hline Poder dar al médico su opinión acerca de su tratamiento & $3,83 \pm 0,94$ \\
\hline Aclaraciones del médico sobre dudas del tratamiento & $3,87 \pm 0,94$ \\
\hline Entendimiento la información que le brinda el médico & $3,87 \pm 0,97$ \\
\hline Tiempo que le dedica el médico en la consulta & $3,87 \pm 0,97$ \\
\hline Satisfacción general con el médico & $3,94 \pm 0,95$ \\
\hline II. Funcionamiento del servicio & $3,71 \pm 0,82$ \\
\hline La frecuencia con que le dan todas las medicinas prescritas en la farmacia de la clínica & $3,60 \pm 0,99$ \\
\hline La comodidad del hospital o la clínica & $3,60 \pm 0,95$ \\
\hline La limpieza del hospital o la clínica & $3,68 \pm 0,94$ \\
\hline La confianza que tiene en el hospital o clínica & $3,76 \pm 0,94$ \\
\hline III. Aspectos administrativos & $3,35 \pm 0,87$ \\
\hline La organización del hospital & $3,56 \pm 0,97$ \\
\hline Los horarios respecto a sus citas asignadas & $3,41 \pm 1,06$ \\
\hline La facilidad que se resuelven los problemas administrativos de su clínica & $3,39 \pm 1,08$ \\
\hline El tiempo de espera desde el momento de su cita hasta que lo pasan a consulta & $2,95 \pm 1,13$ \\
\hline IV. Atención en áreas específicas & $3,78 \pm 0,86$ \\
\hline Atención que proporciona el laboratorio & $3,77 \pm 0,99$ \\
\hline Atención que proporciona la farmacia & $3,72 \pm 1,06$ \\
\hline Atención por parte del servicio de enfermería & $3,82 \pm 1,01$ \\
\hline Atención por parte de la recepción & $3,69 \pm 1,08$ \\
\hline \multirow[t]{2}{*}{ Atención por parte de trabajo social } & $3,67 \pm 1,13$ \\
\hline & $\begin{array}{c}\mathbf{n}=\mathbf{5 5 7} \\
\%\end{array}$ \\
\hline \multicolumn{2}{|l|}{ I. Relación médico-paciente } \\
\hline Satisfecho & 89,2 \\
\hline Insatisfecho & 10,8 \\
\hline \multicolumn{2}{|l|}{ II. Funcionamiento del servicio } \\
\hline Satisfecho & 87,2 \\
\hline Insatisfecho & 12,8 \\
\hline
\end{tabular}




\begin{tabular}{|l|c|}
\hline III. Aspectos administrativos & 67,5 \\
\hline Satisfecho & 32,5 \\
\hline Insatisfecho & \\
\hline IV. Atención en áreas específicas & 85,5 \\
\hline Satisfecho & 14,5 \\
\hline Insatisfecho & \\
\hline
\end{tabular}

Fuente: elaboración propia con datos recabados en dos instituciones públicas de la Ciudad de México

administrativos y $14,5 \%$ con la atención de los diferentes servicios (ver tabla 3 ).

En los resultados del análisis bivariado y del modelo de regresión logística correspondiente a la satisfacción con la relación médico-paciente se observó que entre los hombres, los heterosexuales y bisexuales tuvieron menor probabilidad de insatisfacción $(\mathrm{RM}=0,25$, $\mathrm{IC}=0,09-0,68$ y $\mathrm{RM}=0,38, \mathrm{IC}=0,15-0,98$, respectivamente) (ver tablas 4 y 6 ).

Respecto al funcionamiento del servicio, se observó tanto en población total como entre los hombres que fue más frecuente la insatisfacción en los derechohabientes del ISSSTE respecto a los que eran atendidos en la clínica de la ss-GDF $(\mathrm{RM}=5,12, \mathrm{IC}=2,47-10,5 \mathrm{y}$ $\mathrm{RM}=5,48, \mathrm{IC}=2,30-13,0)($ tablas 4-6). Entre los hombres se observó que los heterosexuales tuvieron menor probabilidad de insatisfacción con el funcionamiento del servicio $(\mathrm{RM}=0,37, \mathrm{IC}=0,15-0,86)($ tabla 6$)$.

En cuanto al índice de aspectos administrativos, en el análisis bivariado se observó mayor prevalencia de insatisfacción entre los hombres, los solteros y las personas con mayor escolaridad, siendo las diferencias estadísticamente significativas (tabla 4). En los modelos de regresión se observó que las personas que únicamente había cursado la primaria tuvieron menos probabilidad de insatisfacción $(\mathrm{RM}=0,46, \mathrm{IC}=0,22-0,96$ en la

136 población total y $\mathrm{RM}=0,37, \mathrm{IC}=0,15-0,92$ entre los hombres) (tablas 5 y 6 ).
Respecto a la atención de las diferentes áreas de la clínica se observó que entre las personas derechohabientes al ISSSTE fue mayor la prevalencia de insatisfacción ( $\mathrm{RM}=3,30, \mathrm{IC}=1,64-6,61$ en la población total y $\mathrm{RM}=2,75, \mathrm{IC}=1,22-6,16$ entre los hombres) (tablas 4-6).

\section{Discusión y conclusiones}

En el presente estudio se encontró que el mayor porcentaje de insatisfacción se presentó con los aspectos administrativos (32,5\%), seguido por el funcionamiento del servicio $(12,8 \%)$; mientras que el aspecto con mejor evaluación fue la relación médico-paciente con $10,8 \%$ de insatisfacción. Esto indica que la mayoría de los participantes estuvieron satisfechos con los servicios de las dos instituciones públicas de atención a personas seropositivas que fueron consideradas en el presente estudio. Lo anterior corresponde a lo observado en otras poblaciones de México. Por ejemplo, entre usuarios de la consulta externa de quince hospitales públicos del estado de Hidalgo la prevalencia global de insatisfacción con la atención recibida fue de $15,1 \%$ (20).

Dentro de los aspectos administrativos el rubro que tuvo menor puntaje en la escala de satisfacción fue el relacionado con el tiempo de espera en pasar a consulta. El largo tiempo de espera es uno de los principales motivos por los que los usuarios perciben como mala la calidad de la atención en ser- 
FACTORES SOCIODEMOGRÁFICOS ASOCIADOS A LA SATISFACCIÓN CON LOS SERVICIOS PÚBLICOS DE SALUD ENTRE PERSONAS CON VIH/SIDA

TABLA 4. RELACIÓN ENTRE LAS CARACTERÍSTICAS SOCIODEMOGRÁFICAS Y LA SATISFACCIÓN DE LOS USUARIOS DE CLÍNICAS DE ATENCIÓN DE VIH

\begin{tabular}{|c|c|c|c|c|c|c|c|c|}
\hline & \multicolumn{6}{|c|}{ Primera escala } & \multirow{2}{*}{\multicolumn{2}{|c|}{$\begin{array}{l}\text { Segunda escala } \\
\text { I1. Atención en } \\
\text { áreas específicas }\end{array}$}} \\
\hline & \multicolumn{2}{|c|}{$\begin{array}{l}\text { I1. Relación } \\
\text { médico-paciente }\end{array}$} & \multicolumn{2}{|c|}{$\begin{array}{l}\text { I2. Funcionamien- } \\
\text { to del servicio }\end{array}$} & \multicolumn{2}{|c|}{$\begin{array}{l}\text { I3. Aspectos } \\
\text { administrativos }\end{array}$} & & \\
\hline & $\mathbf{S}$ & I & $\mathbf{S}$ & I & $\mathbf{S}$ & $\mathbf{I}$ & $\mathbf{S}$ & $\mathbf{I}$ \\
\hline & $\%$ & $\%$ & $\%$ & $\%$ & $\%$ & $\%$ & $\%$ & $\%$ \\
\hline Edad (media) & 36,8 & 34,9 & 36,2 & 39,3 & 36,8 & 36,9 & 36,4 & 37,8 \\
\hline \multicolumn{9}{|l|}{ Sexo } \\
\hline Hombre & 88,2 & 11,8 & 86,4 & 13,6 & 65,2 & $34,8^{*}$ & 84,2 & 15,8 \\
\hline Mujer & 93,6 & 6,4 & 90,7 & 9,3 & 77,1 & 22,9 & 90,8 & 9,2 \\
\hline \multicolumn{9}{|l|}{ Estado civil } \\
\hline Soltero & 88,8 & 11,2 & 87,6 & 12,4 & 64,0 & $36,0^{*}$ & 84,1 & 15,9 \\
\hline Casado-unión libre & 89,7 & 10,3 & 87,7 & 12,3 & 70,5 & 29,5 & 88,4 & 11,6 \\
\hline Divorciado-viudo & 90,6 & 9,4 & 84,4 & 15,6 & 79,7 & 20,3 & 85,9 & 14,1 \\
\hline \multicolumn{9}{|l|}{ Orientación sexual } \\
\hline Heterosexual & 94,6 & $5,4^{*}$ & 87,4 & 12,6 & 68,5 & 31,5 & 85,6 & 14,4 \\
\hline Bisexual & 92,5 & 7,5 & 87,5 & 15,5 & 65,0 & 35,0 & 83,8 & 16,3 \\
\hline Homosexual & 84,0 & 16,0 & 85,5 & 14,5 & 64,1 & 35,9 & 83,6 & 16,4 \\
\hline \multicolumn{9}{|l|}{ Ocupación } \\
\hline No trabaja & 91,5 & 8,5 & 87,3 & $12,7^{* *}$ & 72,5 & 27,5 & 86,6 & $13,4^{*}$ \\
\hline $\begin{array}{l}\text { Trabajador de empresa } \\
\text { privada }\end{array}$ & 89,6 & 10,4 & 87,5 & 12,5 & 70,8 & 29,2 & 90,6 & 9,4 \\
\hline Trabajador de Gobierno & 89,9 & 10,1 & 72,2 & 27,8 & 60,8 & 39,2 & 72,2 & 27,8 \\
\hline $\begin{array}{l}\text { Trabaja para otra } \\
\text { persona }\end{array}$ & 88,7 & 11,3 & 88,7 & 11,3 & 70,4 & 29,6 & 85,9 & 14,1 \\
\hline Trabaja por su cuenta & 87,7 & 12,3 & 93,1 & 6,9 & 64,4 & 35,6 & 88,4 & 11,6 \\
\hline $\begin{array}{l}\text { Empresario, patrón, } \\
\text { empleador o dueño }\end{array}$ & 82,6 & 17,4 & 95,7 & 4,3 & 56,6 & 43,5 & 82,6 & 17,4 \\
\hline \multicolumn{9}{|c|}{ Escolaridad del jefe de familia } \\
\hline Primaria o menos & 94,6 & 5,4 & 94,5 & 5,5 & 78,4 & $21,6^{*}$ & 87,8 & 12,2 \\
\hline Secundaria & 85,9 & 14,1 & 87,3 & 12,7 & 70,4 & 29,6 & 85,2 & 14,8 \\
\hline Bachillerato & 89,3 & 10,7 & 87,4 & 12,6 & 67,4 & 32,6 & 86,5 & 13,5 \\
\hline Licenciatura y más & 89,6 & 10,4 & 82,4 & 17,6 & 57,6 & 42,4 & 82,4 & 17,6 \\
\hline \multicolumn{9}{|l|}{ Institución } \\
\hline ISSSTE & 92,0 & 8,0 & 70,0 & $30,0^{* *}$ & 63,0 & 37,0 & 74,0 & $26,0^{* *}$ \\
\hline SS-GDF & 88,6 & 11,4 & 91,0 & 9,0 & 68,5 & 31,5 & 88,0 & 12,0 \\
\hline
\end{tabular}

Abreviaturas: S: satisfecho; I: insatisfecho. ISSSTE: Instituto de Seguridad y Servicios Sociales de los Trabajadores del Estado. SS-GDF: Secretaría de Salud del Gobierno de Distrito Federal. $* \mathrm{p}<0,05, * * \mathrm{p}<0,001$. Nota: la suma del $100 \%$ es sobre las filas.

Fuente: elaboración propia con datos recabados en dos instituciones públicas de la Ciudad de México 
TABLA 5. FACTORES ASOCIADOS A LA INSATISFACCIÓN CON RELACIÓN MÉdICO-PACIENTE, ORGANIZACIÓN dE LA CLÍNICA Y ATENCIÓN DE LOS DIFERENTES SERVICIOS. TODA LA POBLACIÓN ESTUDIADA

\begin{tabular}{|c|c|c|c|c|c|c|c|c|}
\hline & \multicolumn{2}{|c|}{$\begin{array}{l}\text { I1. Relación } \\
\text { médico-paciente }\end{array}$} & \multicolumn{2}{|c|}{$\begin{array}{l}\text { I2. Funcionamien- } \\
\text { to del servicio }\end{array}$} & \multicolumn{2}{|c|}{$\begin{array}{c}\text { F3. Aspectos } \\
\text { administrativos }\end{array}$} & \multicolumn{2}{|c|}{$\begin{array}{c}\text { F1. Atención en } \\
\text { servicios específicos }\end{array}$} \\
\hline & RM & IC & RM & IC & RM & IC & RM & IC \\
\hline \multicolumn{9}{|l|}{ Edad } \\
\hline 16 a 25 & 1,71 & $0,49-5,90$ & 1,24 & $0,42-3,63$ & 0,98 & $0,47-2,07$ & 1,22 & $0,44-3,36$ \\
\hline 26 a 35 & 1,90 & $0,61-5,88$ & 1,07 & $0,42-2,68$ & 0,81 & $0,42-1,58$ & 1,23 & $0,51-2,99$ \\
\hline 36 a 50 & 1,56 & $0,54-4,53$ & 0,99 & $0,46-2,13$ & 0,78 & $0,43-1,44$ & 1,22 & $0,57-2,64$ \\
\hline 51 y más & 1,00 & & 1,00 & & 1,00 & & 1,00 & \\
\hline \multicolumn{9}{|l|}{ Sexo } \\
\hline Hombres & 1,92 & $0,80-4,63$ & 1,47 & $0,66-3,27$ & 1,32 & $0,77-2,26$ & 1,82 & $0,84-3,93$ \\
\hline Mujeres & 1,00 & & 1,00 & & 1,00 & & 1,00 & \\
\hline \multicolumn{9}{|l|}{ Estado civil } \\
\hline Soltero & 0,77 & $0,28-2,10$ & 0,76 & $0,31-1,82$ & 1,97 & $0,94-3,94$ & 1,09 & $0,46-2,59$ \\
\hline Casado-unión libre & 0,82 & $0,29-2,31$ & 0,69 & $0,27-1,72$ & 1,58 & $0,75-3,30$ & 0,72 & $0,29-1,81$ \\
\hline Divorciado-viudo & 1,00 & & 1,00 & & 1,00 & & 1,00 & \\
\hline \multicolumn{9}{|c|}{ Escolaridad del jefe de familia } \\
\hline Primaria o menos & 0,58 & $0,16-1,99$ & 0,58 & $0,17-1,97$ & 0,46 & $0,22-0,96$ & 1,36 & $0,52-3,50$ \\
\hline Secundaria & 1,42 & $0,62-3,24$ & 1,57 & $0,69-3,53$ & 0,63 & $0,36-1,11$ & 1,59 & $0,74-3,39$ \\
\hline Bachillerato & 0,98 & $0,46-2,11$ & 1,14 & $0,57-2,25$ & 0,68 & $0,42-1,10$ & 1,08 & $0,56-2,08$ \\
\hline Licenciatura y más & 1,00 & & 1,00 & & 1,00 & & 1,00 & \\
\hline \multicolumn{9}{|l|}{ Institución } \\
\hline ISSSTE & 0,85 & $0,33-2,14$ & 5,12 & $2,47-10,5$ & 1,10 & $0,63-1,93$ & 3,30 & $1,64-6,61$ \\
\hline SS-GDF & 1,00 & & 1,00 & & 1,00 & & 1,00 & \\
\hline
\end{tabular}

Abreviaturas: RM: razón de momios; IC: intervalo de confianza. ISSSTE: Instituto de Seguridad y Servicios Sociales de los Trabajadores del Estado. Ss-GDF: Secretaría de Salud del Gobierno de Distrito Federal.

Fuente: elaboración propia con datos recabados en dos instituciones públicas de la Ciudad de México

vicios ambulatorios de salud en México (21). Dicha situación puede influir, entre otros aspectos, en la asistencia de las personas a las instituciones de salud. En México, mediante la Encuesta Nacional de Salud y Nutrición 2012 se observó que el tiempo de espera prolongado fue la principal razón por la cual las personas no regresarían a la institución de salud (pública o privada) en donde fueron atendidos en las dos semanas previas (22). Más recientemente se ha reportado que un menor tiempo de espera dentro de los servicios de salud está asociado a una percepción de la mejoría en salud después de la atención recibida (23).

En el presente trabajo se observó que las personas que tenían la primaria como grado de escolaridad tuvieron menor probabilidad de insatisfacción con los aspectos administrativos. Este resultado coincide con otros estudios. En un metanálisis sobre la satisfacción de los pacientes con la atención médica, se reportó que las personas con menor escolaridad tuvieron mayor satisfacción con la atención médica (24) y en un estudio reciente realizado en España se identificó 
FACTORES SOCIODEMOGRÁFICOS ASOCIADOS A LA SATISFACCIÓN CON LOS SERVICIOS PÚBLICOS DE SALUD ENTRE PERSONAS CON VIH/SIDA

TABLA 6. FACTORES ASOCIAdOS A LA INSATISFACCIÓN CON RELACIÓN MÉdICO-PACIENTE, ORGANIZACIÓN DE LA CLÍNICA Y ATENCIÓN DE LOS DIFERENTES SERVICIOS. HOMBRES.

\begin{tabular}{|c|c|c|c|c|c|c|c|c|}
\hline & \multicolumn{2}{|c|}{$\begin{array}{l}\text { F1. Relación } \\
\text { médico-paciente }\end{array}$} & \multicolumn{2}{|c|}{$\begin{array}{l}\text { F2. Funcionamien- } \\
\text { to del servicio }\end{array}$} & \multicolumn{2}{|c|}{$\begin{array}{l}\text { F3. Aspectos } \\
\text { administrativos }\end{array}$} & \multicolumn{2}{|c|}{$\begin{array}{l}\text { F1. Atención en ser- } \\
\text { vicios específicos }\end{array}$} \\
\hline & RM & IC & RM & IC & вм & IC & RM & IC \\
\hline \multicolumn{9}{|l|}{ Edad } \\
\hline 16 a 25 & 1,05 & $0,28-3,89$ & 0,95 & $0,29-3,04$ & 0,95 & $0,41-2,21$ & 0,76 & $0,25-2,30$ \\
\hline 26 a 35 & 1,29 & $0,39-4,22$ & 0,81 & $0,29-2,21$ & 0,94 & $0,44-2,00$ & 0,93 & $0,36-2,37$ \\
\hline 36 a 50 & 1,09 & $0,36-3,27$ & 0,83 & $0,36-1,89$ & 0,89 & $0,45-1,75$ & 0,96 & $0,42-2,15$ \\
\hline 51 y más & 1,00 & & 1,00 & & 1,00 & & 1,00 & \\
\hline \multicolumn{9}{|l|}{ Estado civil } \\
\hline Soltero & 0,42 & $0,13-1,31$ & 0,50 & $0,18-1,39$ & 1,78 & $0,73-4,32$ & 0,82 & $0,31-2,19$ \\
\hline Casado-unión libre & 0,51 & $0,15-1,71$ & 0,61 & $0,21-1,78$ & 1,64 & $0,65-4,09$ & 0,58 & $0,20-1,65$ \\
\hline Divorciado-viudo & 1,00 & & 1,00 & & 1,00 & & 1,00 & \\
\hline \multicolumn{9}{|c|}{ Escolaridad del jefe de familia } \\
\hline Primaria o menos & 0,83 & $0,20-3,31$ & 0,68 & $0,17-2,64$ & 0,37 & $0,15-0,92$ & 1,51 & $0,54-4,23$ \\
\hline Secundaria & 1,74 & $0,71-4,22$ & 1,27 & $0,53-3,05$ & 0,63 & $0,34-1,17$ & 1,57 & $0,71-3,48$ \\
\hline Bachillerato & 1,32 & $0,59-2,95$ & 1,09 & $0,53-2,25$ & 0,80 & $0,48-1,33$ & 1,02 & $0,51-2,03$ \\
\hline Licenciatura y más & 1,00 & & 1,00 & & 1,00 & & 1,00 & \\
\hline \multicolumn{9}{|l|}{ Institución } \\
\hline ISSSTE & 1,04 & $0,36-2,98$ & 5,48 & $2,30-13,0$ & 1,39 & $0,73-2,65$ & 2,75 & $1,22-6,16$ \\
\hline SS-GDF & 1,00 & & 1,00 & & 1,00 & & 1,00 & \\
\hline \multicolumn{9}{|l|}{ Orientación sexual } \\
\hline Heterosexual & 0,25 & $0,09-0,68$ & 0,37 & $0,15-0,86$ & 0,81 & $0,46-1,42$ & 0,64 & $0,30-1,37$ \\
\hline Bisexual & 0,38 & $0,15-0,98$ & 0,95 & $0,43-2,12$ & 1,13 & $0,65-1,95$ & 0,98 & $0,48-1,99$ \\
\hline Homosexual & 1,00 & & 1,00 & & 1,00 & & 1,00 & \\
\hline
\end{tabular}

Abreviaturas: RM: razón de momios; IC: intervalo de confianza. ISSSTE: Instituto de Seguridad y Servicios Sociales de los Trabajadores del Estado. Ss-GDF: Secretaría de Salud del Gobierno de Distrito Federal.

Fuente: elaboración propia con datos recabados en dos instituciones públicas de la Ciudad de México

que las personas que tenían la primaria como grado de escolaridad tuvieron mayor probabilidad de satisfacción con la asistencia hospitalaria (25).

Cuando se han utilizado otros indicadores de posición socioeconómica, se ha observado la misma tendencia. Por ejemplo, en un estudio realizado en México (20), los usuarios que estaban insatisfechos con la atención en las unidades de salud tenían un mayor ingreso económico. De acuerdo con lo anterior, es probable que las personas que tienen una mejor posición socioeconómica tengan mayores expectativas respecto a la atención que reciben en las instituciones de salud.

En este estudio se encontró que entre los hombres y las personas solteras existió mayor prevalencia de insatisfacción con los aspectos administrativos. Este resultado coincide con lo reportado en un estudio realizado en personas con VIH/SIDA atendidas en siete instituciones de salud de Vietnam (17), en el 
que entre los hombres y las personas solteras existió mayor prevalencia de insatisfacción con la atención recibida en la institución de salud a la cual asistían.

En cuanto al funcionamiento del servicio, el rubro que tuvo menor puntaje en la escala de satisfacción fue la frecuencia con que los usuarios reciben todas las medicinas prescritas en la farmacia de la clínica. Dicho resultado contrasta con el compromiso asumido por el Gobierno mexicano de asegurar el acceso al TAR a todas las personas con VIH. Esto es preocupante pues la adherencia al tratamiento depende, entre otros factores, del acceso a los medicamentos, ya que estos son un aspecto clave para el control de la infección y detener la progresión de la enfermedad.

En comparación con las personas atendidas en la Clínica Especializada Condesa (Ss-GDF), los participantes derechohabientes de ISSSTE tuvieron mayor probabilidad de estar insatisfechos con el funcionamiento del servicio así como con la atención en las diferentes áreas de la clínica. Probablemente, la mejor calificación de la clínica especializada refleja la particularidad de estar enfocada a una enfermedad y que se cuenta con personal de diferentes áreas con más experiencia, mientras que ese grado de integración y experiencia puede no ser alcanzado en un servicio que forma parte de un hospital con múltiples especialidades. Aunque también es posible que las personas que son atendidas en la Clínica Condesa consideren que por el hecho de ser atendidas en un servicio "público y gratuito" no pueden exigir un mejor servicio; mientras que los derechohabientes del ISSSTE pueden percibir que sus aportaciones a la seguridad social les dan el derecho a exigir servicios con mayor calidad y por ello sus expectativas sean mayores.

Respecto a la relación médico-paciente, los rubros que tuvieron menor puntaje en la escala de satisfacción fueron los referentes a la información que el médico otorga sobre los efectos secundarios del tratamiento, la exploración física que realiza y la posibilidad de que las personas le expresen su opinión acerca del tratamiento. La información que el médico da respecto al tratamiento o la enfermedad ha sido uno de los predictores de satisfacción reportado en estudios de México y otros países. En México, en usuarios de consulta externa de hospitales públicos (20), una de las variables relacionadas con la insatisfacción de la atención fue la ausencia de información respecto al padecimiento de la persona.

En personas con enfermedades crónicas atendidas en instituciones de seguridad social, las calificaciones más bajas dentro del índice de satisfacción con la relación médico-paciente fueron en los ítems "el médico permite la opinión del paciente sobre los tratamientos" y "la comprensión de la información” (26). Además, en un estudio cualitativo realizado en pacientes hospitalizados en una institución de tercer nivel, se identificó que los pacientes identificaban como inadecuado el trato cotidiano que recibían tanto del personal médico como de enfermería, particularmente porque no percibían la suficiente confianza para solicitar información respecto a su enfermedad (27). Estos hallazgos son consistentes con los reportados en países de alto ingreso.

En individuos que acudían a una clínica de salud de Estados Unidos, se observó que uno de los predictores de la satisfacción con la relación médico-paciente fue la información que el médico otorgaba respecto a la causa y los síntomas de la enfermedad, así como la duración de esta (28). En personas que habían recibido atención en un hospital en España, el recibir información sobre su estado de salud fue uno de los predictores de satisfacción con la asistencia hospitalaria (25). 
En particular, en pacientes con viH que recibían atención primaria en dos clínicas de Houston, Texas, el mayor predictor de la satisfacción global con la atención recibida fue la satisfacción con la relación médicopaciente (7).

En el presente estudio, los hombres heterosexuales y bisexuales tuvieron una menor probabilidad de insatisfacción con la relación médico-paciente y con el funcionamiento del servicio en comparación con los homosexuales. Es interesante notar que la satisfacción fue menor en el grupo de hombres homosexuales. En México los varones homosexuales han participado activamente para que las personas seropositivas cuenten con acceso a servicios médicos y acceso al TAR. Se puede hipotetizar, entonces, que los hombres homosexuales perciben como un derecho exigible la atención médica de la infección por VIH y por ello tienen mayores expectativas respecto al trato que reciben.

Por el contrario, los grupos con menor insatisfacción (i. e.. hombres heterosexuales, personas casadas y mujeres) son aquellos que simbólicamente están menos relacionados con la infección por viH (29). Por ello, es probable que las personas de estos últimos grupos experimenten más estigma por la enfermedad (pues culturalmente no se espera que sean afectados) y que esa condición influya en que sus estándares para calificar la atención recibida sean inferiores.

Entre las limitaciones del estudio se encuentra el hecho de que no se utilizó muestreo probabilístico, lo cual limita la posibilidad de generalizar nuestros hallazgos a todas las personas con VIH de la ciudad. Además, aunque incluimos a una institución de seguridad social, no tuvimos acceso a personas de la principal institución de seguridad social de México (i. e., IMSS). Sin embargo, debe notarse que nuestra muestra es heterogénea en términos sociodemográficos, lo cual permitió identificar algunos patrones relevantes. Además, en los dos centros de atención todas las personas fueron invitadas a participar durante el trabajo de campo.

En conclusión, la mayoría de las personas con VIH están satisfechas con la atención recibida en instituciones públicas de salud de la Ciudad de México. Sin embargo, en este trabajo se identificaron aspectos que pueden ser mejorados. Además, ciertos grupos están menos satisfechos con los servicios de las instituciones en donde reciben atención y tratamiento, lo cual puede ser producto de diferencias en el trato recibido o bien cómo este es percibido por las personas. Para que la percepción de la calidad de los servicios mejore, uno de los principales aspectos que considerar son las experiencias reportadas por los pacientes respecto a la atención recibida en las instituciones de salud.

Un primer resultado que puede retroalimentar a los servicios y especialmente al personal médico, es el hecho de que entre los rubros que tuvieron la calificación más baja se encuentran "la información que el médico otorga sobre los efectos secundarios de los medicamentos" y "el poder dar opinión acerca del tratamiento". Esto pone en evidencia la necesidad de que los modelos de atención deben modificarse de modo que los profesionales de la salud conduzcan la consulta propiciando la participación activa de las personas, además de que estas puedan tomar decisiones informadas sobre su tratamiento, lo cual puede promover mayor apego a este. El desabasto de los medicamentos y el tiempo de espera fueron otros aspectos identificados que requieren mejora.

Ante esto, es importante que las instituciones de salud realicen modificaciones de tal forma que los servicios y el flujo de pacientes sean manejados de una forma conveniente con 
el objetivo de simplificar y hacer eficientes los procedimientos administrativos para reducir el tiempo de espera y garantizar la disponibilidad y acceso a la TAR a las personas con VIH. En el futuro convendría confirmar nuestros hallazgos en muestras representativas de pacientes con ViH. También es necesario evaluar la correspondencia de los reportes proporcionados por las personas con indicadores del funcionamiento de los servicios; por ejemplo, conviene documentar si la percepción de las deficiencias en la administración tiene relación con el funcionamiento inadecuado de los procedimientos institucionales. Finalmente, el siguiente paso es diseñar programas encaminados a mejorar los aspectos más problemáticos aquí identificados y evaluar si dichos programas inciden positivamente en la satisfacción de las personas con los servicios.

\section{Referencias bibliográficas}

1. ONUSIDA. Informe mundia.l Informe de ONUSIDA sobre la epidemia mundial de sida 2012 [internet] [acceso: 15 de junio de 2012]. Disponible en: http://www.unaids.org/en/media/unaids/ contentassets/documents/epidemiology/2012/ gr2012/20121120_UNAIDs_Global_Report_2012_ with_annexes_es.p̄pdf.

2. Centro Nacional para la Prevención y el Control del VIH/SIDA (CENSIDA). Vigilancia epidemiológica de casos de VIH/SIDA en México. Registro Nacional de Casos de SIDA. Actualización preliminar hasta la semana 52 del 2012. 2012 [internet] [acceso: 10 de julio de 2012]. Disponible en: http://www. censida.salud.gob.mx/descargas/2012/sida_vih30sept2012pub.pdf.

3. World Health Organization. Quality of care. Process for makin g strategic choices in health systems. Ginebra, Suiza 2006.

4. Lochman JE. Factors related to patients' satisfaction with their medical care. J Community Health. 1983; 9 (2): 91-109.

5. Ware JE, Snyder MK, Wright WR, Davies AR. Defining and measuring patient satisfaction with medical care. Eval Program Plann. 1983; 6: 247-63.

6. Mpinga EK, Chastonay P. Satisfaction of patients: a right to health indicator? Health Policy. 2011; 100: 144-50.

7. Dang BN, Westbrook RA, Rodríguez-Barradas MC, Giordano TP. Identifying drivers of overall sa- tisfaction in patients receiving HIV primary care: A cross-sectional study. Plos One 2012; 7 (8): e42980.

8. Kincey J, Bradshaw P. Patients satisfaction and reported acceptance of advice in general practice. J R Coll Gen Pract. 1975; 25 (157): 558-66.

9. Becker MH, Maiman LA. Strategies for enhancing patient compliance. J Community Health. 1980; 6 (2): 113-35.

10. Xiao H, Barber JP. The effect of perceived health status on patient satisfaction. Value Health. 2008; 11 (4): 719-25.

11. Sayles JN, Wong MD, Kinsler JJ, Martins D, Cunningham WE. The association of stigma with selfreported access to medical care and antiretroviral therapy adherence in persons living with HIV/AIDS. J Gen Intern Med. 2009; 24 (10): 1101-8.

12. Del Pilar Lavielle-Sotomayor M, Cerón-Ruiz M, Tovar-Larrea P, Bustamante-Rojano JJ. Satisfacción con la atención a la salud. Rev Med IMSS. 2008; 46 (2): 129-34.

13. Ramírez de la-Roche O, López-Serrano A, Barragán-Solis A, E. A-A. Satisfacción del usuario en una unidad de medicina familiar de una institución de seguridad social de la ciudad de México. Arch Med Fam. 2005; 7: 22-6.

14. González-Castillo BI, López-Rojas P, MarínCotoñieto IA, Haro-García L, Macías-Rangel A. Satisfacción del usuario de los servicios de salud en el trabajo. Rev Med IMSS. 2002; 40( 4): 301-4.

15. Bronfman-Pertzovsky MN, López-Moreno S, Magis-Rodríguez C, Moreno-Altamirano A, Rutstein S. Prenatal care at the first level of care: characteristics of providers that affect users' satisfaction. Salud Pública Mex. 2003; 45 (6): 445-54.

16. Vladislavovna-Doubova SV, Pérez-Cuevas R, Zepeda-Arias M, Flores-Hernández S. Satisfaction of patients suffering from type 2 diabetes and/or hypertension with care offered in family medicine clinics in Mexico. Salud Publica Mex. 2009; 51 (3): 231-9.

17. Tran BX, Nguyen NP. Patient satisfaction with HIV/ AIDS care and treatment in the decentralization of services delivery in Vietnam. Plos One. 2012;7 (10): e46680.

18. Centro Nacional para la Prevención y el Control del VIH/SIDA (CENSIDA). El VIH/SIDA en México. México: Centro Nacional para la Prevención y el Control del VIH/sIDA; 2008.

19. Rattray J, Jones MC. Essential elements of questionnaire design and development. J Clin Nurs. 2007; 16 (2): 234-43.

20. Ortiz-Espinosa RM, Muñoz-Juárez S, TorresCarreño E. Satisfacción de los usuarios de 15 hospitales de Hidalgo, México. Rev Esp Salud Pública. 2004; 45: 527-37.

21. Ramírez-Sanchez TJ, Najera-Aguilar P, NigendaLopez G. Perception of the quality of care in the health services in Mexico: perspective of the users. Salud Pública Mex 1998; 40 (1): 3-12.

22. Instituto Nacional de Salud Pública. Evidencia para la política pública en salud. Calidad de los procesos de atenciónambulatoria en los servicios de salud: estándares y mejora organizacional. México 2012 
[internet]. Disponible en http://ensanut.insp.mx/ doctos/analiticos/CalidadAtencion.pdf

23. Reyes-Morales H, Flores-Hernández S, SaucedaValenzuela A, Vértiz-Ramírez J, Juárez-Ramírez C, Wirtz V et ál. Percepción de los usuarios sobre la calidad de la atención ambulatoria en servicios de salud en México. Salud Pública Mex 2013; 55 (supl 2): S100-S5.

24. Hall JA, Dornan MC. Patient sociodemographic characteristics as predictors of satisfaction with medical care: a meta-analysis. Soc Sci Med. 1990; 30 (7): 811-8.

25. Dierssen-Sotos T, Rodríguez-Cundin P, RoblesGarcía M, Brugos-Llamazares V, Gomez-Acebo I, Llorca J. Factors associated with patient satisfaction with hospital care. An Sist Sanit Navar. 2009; 32 (3): 317-25.
26. Vladislavovna-Doubova S, Pérez-Cuevas R, ReyesMorales H. Autopercepcion del estado de salud en climatericas derechohabientes del Instituto Mexicano del Seguro Social. Salud Publica Mex. 2008; 50 (5): 390-6.

27. Herrera-Kiengelher L, Villamil-Álvarez M, Pelcastre-Villafuerte B, Cano-Valle F, López-Cervantes M. Relationship between health providers and patients in Mexico City. Rev Saude Publica. 2009; 43 (4): 589-94.

28. Jackson JL, Chamberlin J, Kroenke K. Predictors of patient satisfaction. Soc Sci Med. 2001; 52 (4): 609-20.

29. Rodríguez-Martínez EN, García-Gaviria N. Enfermedad y significación: Estigma y monstruosidad del VIH/SIDA. Opción. 2006; 22 (50): 9-28. 